schen hohen Investitionen in die wissenschaftliche Forschung (2 bis $4 \%$ ) und dem sozioökonomischen Entwicklungs- und Lebensstandard in den OECDLändern, sowie an der offenkundigen Bewunderung für die Asiaten, wenn es um deren schnelles Wachstum, deren neue internationale Führungsrolle und die Überwindung der Armut geht. Was können wir uns von den lateinamerikanischen Ländern erhoffen, die wissenschaftlich das höchste Niveau vertreten (Argentinien, Brasilien, Kolumbien und Mexiko). Schon vor Jahrzehnten versprachen die Machthaber dort, $1 \%$ des BIP in die Forschung zu investieren - ohne zu liefern. Diese Vernachlässigung der wissenschaftlichen Entwicklung zu korrigieren ist nicht nur Sache von Spezialisten, die in den letzten Jahren aus Protest auf die Straßen von Buenos Aires, Rio de Janeiro, São Paulo oder Mexiko-Stadt gegangen sind, um sich gegen die massiven Kürzungen des Budgets zu wehren. Wie bei Entscheidungen über das Internet und über die Rolle der Öffentlichkeit in der Bildung, der Wissenschaft und der Gesundheitsversorgung wird diese Frage nun zu einer entscheidenden Verantwortung der Bürger, da sie ebenso wichtig für Lehrer, Wissenschaftler, Studierende, wie für verarmte Familien, Migranten und Arbeitnehmer ist.

\title{
Was sind die Menschen?
}

Aktuelle Trends stellen diese Frage mit unterschiedlichen Perspektiven. Wir haben intellektuelle Abhandlungen, wie die von Foucault, Lévi-Strauss und Harari gesehen, sowie die Überlegungen soziokultureller Bewegungen, die sozial beeinträchtigt werden und von Entbürgerlichung betroffen sind, wie in lateinamerikanischen Diktaturen, und extreme Konfrontationen mit den Grenzen des Menschlichen durch den Femizid, Leichenraub oder deren bewusstes Verschwindenlassen. Da manche diese Erfahrungen unerträglich finden, sprechen sie den Tätern ihre Menschlichkeit ab und fordern die Todesstrafe. Auf der anderen Seite, die manchmal mit der ersten Perspektive verbunden ist, kommt es durch die Entführung von Daten und der Entscheidungsmacht der Menschen, die von so vielen freiwillig über die Delegierung an algorithmische Systeme ausgeht, zu einem Verschwimmen der Identität und der Grenzen des Menschlichen, die durch die Modernität neu gesetzt werden.

Die Geschichte der kolonialen Eroberungen ist zeitgleich die Geschichte über die Zweifel an der eigenen Identität: von den anderen und denen, die kolonialisieren. Haben die Indios auch eine Seele? Nach Jahren sehen wir, dass 
die gleiche Frage immer noch auftaucht: Mit welchem Recht unterwerfen wir Westler die andersartigen, wo wir selbst unter uns so unterschiedlich sind? Handelt es sich bei afrikanischen Masken und antiken Vasen aus Amerika um Kunst? Kurz darauf heißt es dann, die Museen der schönen Künste, der modernen oder zeitgenössischen Kunst seien unvollständig, würden die nichtwestlichen Werke in ihnen fehlen. Wir wissen, wie oft die Antworten, die in dieser schwankenden Diskussion vorherrschen aus Imperien stammen, die den Widerstand ersticken. Wir wissen, dass die prominenten Stimmen in solchen Diskussionen von denen kommen, die Revolutionen institutionalisieren, sogar die technologischen, die von Monopolen für bestimmte Zwecke entwendet werden. Die heutige Zeit ist keine Ausnahme. Vielleicht besteht ihre Neuartigkeit darin, dass viele Staatsbürgerschaften das de-programmieren, was die Souveränität des Imperiums, und jetzt die Algorithmen, einmal zu befehlen schienen. Die destabilisierenden Fragen kommen aus vielen Richtungen. Millionen von amerikanischen Indigenen, Asiaten und Afrikanern, die feministischen Bewegungen und die LGBT-Bewegungen tragen dazu bei, dass die Frage nach dem Menschlichen neu geschrieben wird. Was sind Bürger*innen?

Die radikale Dekonstruktion des liberalen Menschenbildes tangiert die Prinzipien der Demokratien und der historischen Linken: Was bedeutet die Verteidigung der Rechte? Wie können diese aussehen und wie können sie durch das Handeln von Menschenrechtsorganisationen und Bürgern im Kampf für ihre Rechte gestaltet werden? Diejenigen, die weder Zweifel an der »natürlichen Ordnung« haben, noch an der Existenz von nur zwei Geschlechtern, ihrer Nation, Religion oder der eigenen Ethnie, die sie für auserwählt halten, fühlen, sich nicht konzeptionell verunsichert. Von den Ansprüchen auf Staatsbürgerschaft, die Ausländer stellen, zeigen sie sich jedoch erschüttert. In der euphorischen Zeit der Globalisierung war die Akzeptanz pluralistischer Politik die Ressource der Demokraten, die die Diversität wertschätzen wollten, ohne die Synthese der mestizaje (Mestizisierung) auf nationaler Ebene, strikten Optionen des Geschlechts oder kurzum die Dominanz der Stärkeren aufzuerlegen. Die Vereinten Nationen, die UNESCO und nationale Einrichtungen oder NGOs betreiben immer noch Sozialpolitik und fördern kulturelle Werte, die auf diesem pluralistischen Weltbild basieren. Ihre sehr begrenzten Auswirkungen stehen im Kontrast zur Größe des Durcheinanders.

Auch die willkürliche Auswahl der menschlichen Eigenschaften durch die Unternehmen, die die transnationale Konzentration der Märkte anführen - 
also die 50 Konzerne mit den höchsten Einnahmen, Facebook und andere Datensammler und Algorithmus-Riesen - treffen Vereinbarungen über dauerhafte Muster des interkulturellen Zusammenlebens.

Genau wie die Frage »Was ist der Mensch?« noch nicht universell oder homogen beantwortet werden kann, gibt es bisher nur wenig freiwillige Erkenntnisse zur Pluralität. Es ist verständlich, dass die Absicht der MultikultiPolitik oder des interkulturellen Dialogs in der Politik eine friedliche Gouvernementalität über die Welt ist, die nicht selbstzerstörerisch ist. Durch das Fehlen globaler Gremien und fester Übereinkünfte, die Regeln und Sanktionen zur Sicherstellung solcher Richtlinien vorsehen, und die harte Konkurrenz finanzgetriebener Volkswirtschaften, die das Volk längst vergessen haben, wird es allerdings schwierig, dem Pluralismus Raum zu geben.

An dieser Stelle werden wir mit dem Verlust der Subjekte und des Sinns konfrontiert. Seit Marx und den sozialen Bewegungen des 19. und des frühen 20. Jahrhunderts bemühten sich die Sozialwissenschaften um eine Überwindung des Abstrakten hin zum Konkreten, beziehungsweise um eine Wende von der spekulativen Philosophie zum empirischen Wissen über soziale Klassen und Nationalstaaten und die Vorstellungen, die über sie konstruiert werden können. Es ging auch darum, Herrschaftsstrukturen von Unternehmen $\mathrm{zu}$ analysieren und Formen des Widerstands mit strategischem Wert herauszuarbeiten. Der Beitrag der Sozialwissenschaften zum Verständnis der Rolle der Bürger in Nationen und innerhalb des Industriekapitalismus, sowie zur Entzifferung der Erfolge und Misserfolge emanzipatorischer Prozesse, verlieren in Zeiten der finanziellen und der algorithmischen Abstraktion ihre Gültigkeit. Wo ist der Platz in der Gesellschaft für Subjekte, die von sinnrelativierenden Mächten enteignet wurden?

Die gefährdeten Identitäten bzw. Abstraktionen, die die Realität »gewinnen«, erscheinen als möglicher Schutzwall vor der digitalen Globalisierung. Ihre knappen Fortschritte machen auch in wirtschaftlicher und in technologischer Hinsicht deutlich, was die Anthropologie bereits seit Jahren aussagt: Vielmehr als essentielle und selbstkonsistente Identitäten gibt es Wege, um uns selbst zu identifizieren und um herauszufinden, wo wir gerne hingehören möchten, mit wem es sich anzufreunden lohnt und von wem wir uns differenzieren müssen. Deshalb sind die Resultate dieser neuen Abstraktionen unbeständig und von geringer Reichweite.

Durch die Expansion der sozialen Netzwerke, denen jeder angehören kann, erfahren wir auf schwindelerregende Weise von den zahlreichen Arten des Menschseins und von den vielfältigen Entfaltungsmöglichkeiten der ei- 
genen Identität: Wir benutzen mehrere Reisepässe, denken in verschiedenen Sprachen, haben einen Familienteil im einen, und den Rest der Verwandtschaft in zwei anderen Ländern, können wählen zwischen verschiedenen sexuellen Orientierungen, und kombinieren aus einer Fülle an heterogenen Möglichkeiten unsere Kleidung, das Essen, die Inneneinrichtung zu Hause. Meine Suche nach Anerkennung teilt sich unter mehreren anderen Suchen auf.

Diese Streuung der Koexistenz erledigt sich nicht einfach durch die Erweiterung der Fragestellung nach Genderoptionen, der sexuellen Orientierung oder der Zugehörigkeit zu verschiedenen Kulturen. Gegenwärtige Kritiker kommen zu dem Schluss, dass die Schwierigkeit einer Festlegung universell gültiger Definitionen damit einhergeht, dass wir in so genannten Gesellschaften der Desidentifikation leben. Aus dem Blickwinkel eines multikulturellen Fensters befinden wir uns in einer Zeit der mehrfachen Identifikationsmöglichkeiten. Im Zusammenhang mit unserem Thema bedeutet das die Notwendigkeit der Erkenntnis, dass wir zwischen Entbürgerlichung und neuen Formaten der Staatsbürgerschaft schwanken.

Politiker und Wirtschaftswissenschaftler warnen vor den Risiken der Desintegration. Es ist ungewiss, wer die Entscheidungen trifft, noch von welcher Dauer sie sind. Auch ist unklar, wer uns einen Platz oder ein Gehalt auf dem Arbeitsmarkt zuweist, wer uns schadet und gegen wen wir uns auflehnen sollen, wer sich über die Medien und die Netzwerke an uns richtet. Die Unsicherheit wird also verstärkt, wenn die algorithmischen Kreisläufe uns unsere Persönlichkeit abnehmen. Die weitreichende, marketingorientierte Nutzung des algorithmischen Wissens erzeugt Illusionen über unsere Gruppenzugehörigkeit als Nutzer und führt zu Enttäuschungen bei dem Versuch, unsere Rechte geltend zu machen.

Ist das Anwenden anderer Kategorien, wie die der Klasse, für unsere Situierung in dieser Periode der verschärften Ungleichheit noch nützlich? Den traditionellen Parteien, sogar den US-Demokraten, scheint die Umverteilung wieder wichtiger zu werden. In manchen Fällen bereichert sich die rechtsgerichtete Politik, etwa unter Trump, der Front National von Le Pen, und Bolsonaro in Brasilien an dem Unbehagen in der Bevölkerung, das durch die Ungleichheit und die Ausräumung der Rechte erzeugt wird. Gleichzeitig beschuldigen sie diffuse Feindbilder mit gemischten Identitäten: Ausländer, Homosexuelle, Atheisten, Intellektuelle, die Linke und eben diejenigen, die aus der nationalistisch geprägten Kulisse herausstechen. 
Die politische Auseinandersetzung begünstigt einander ähnelnde Vereinfachungen zwischen denjenigen, die mit kritischen Positionen tönen: die Partei Podemos sprach von »der Kaste«, López Obrador von der hochnäsigen »FifiPresse«. Infolgedessen verschwimmen die ursprünglichen Traditionen des Klassenkampfs - Arbeiter gegen Arbeitgeber, Angestellte gegen Regierungen, Bürger gegen das Militär - zu verschiedensten Feindbildern von denen wir den Eindruck haben, dass sie uns verspotten. In einigen Auseinandersetzungen bleibt der Hass gegenüber den Reichen und den einkommensstarken Politikern zusammen mit Motivationen und Taktiken bestehen, die eine Vernetzung des Widerstands schwierig machen: Ebenso wie sich die Gelbwesten von den Großstädtern belächelt fühlen, weil für sie das Benzin zu teuer oder das nächste Krankenhaus zu weit weg ist, führen die Ungleichheiten in Lateinamerika dazu, dass die Einwohner der Hauptstädte, die Banken, die Börsenspekulanten oder Investitionsmanager, oder ehemalige lokale Widersacher zum Sündenbock werden. Ohne eine integrierte Perspektive, in der eine Kombination aus konkreten Verantwortlichen und die Verbindungen zwischen Kartellen, Unternehmen und Politikern genau benannt werden, bleibt die oft kritisierte »Macht« nichts weiter als ein anonymer Slogan. Die Erfahrungen der letzten Jahre hinterlassen bei den Bürgern den Eindruck, dass die Verhaftung irgendeines Präsidenten (darunter drei in Peru, von denen sich einer das Leben nahm), eines lokalen Beteiligten am Odebrecht-Skandal oder eines Drogenbosses möglich ist, aber dass die Netzwerke und der Gesamtapparat der Korruption versteckt und aktiv bleiben. Wo kann man das melden, welches Beschwerdetelefon rufen wir an, um unsere Menschenrechte zu verteidigen?

Während der soziale Sinn an so vielen rätselhaften Fronten zerbröckelt, an denen es (zumindest aus Sicht der Wohlhabenden, der emanzipatorischen Bewegungen, der Philosophie und der modernen Sozialwissenschaft) wenig Interesse für den Sinn zu geben scheint, müssen wir die Überlegungen von Kant und den Begründern des liberalen Gedankens, ja sogar die Ricœurs Frage, erweitern: Was ist die Bedeutung des Sinns? Es geht mir nicht darum, diese Fragen zu verwerfen, sondern sie im Kontext dieser zum Ausweichen tendierenden Zeit neu zu denken. Heute neigen wir dazu, dass Investitionen in die Zeit von kurzer Dauer sind, wie beispielsweise Tweets oder Chats, die wir heute verfassen und in der darauffolgenden Woche löschen. Oder vielleicht sind diese Erinnerungen nicht nur Passagiere in unserem Kopf, sondern sammeln sich in unserem persönlichen Speicher, statt nur auf der Festplatte oder in der Cloud. Deshalb sind wir so abhängig von der Frage, wohin 
das alles führt - nicht nur bei unserer Lieblingsserie, sondern auch in der Realität. Gibt es Ende mit positivem Ausgang?

Gayatri Spivak argumentierte, dass wir uns angesichts der Heterogenität der Konflikte in der »doppelten Abhängigkeit der Emigranten« schulen müssen. Ich würde dieses Argument durch die zahlreicheren Verbindungen der jungen Generation und der simultanen Angehörigen verschiedener Kulturen, Medien und Netzwerke ergänzen. Spivak schlug demnach vor, »unter widersprüchlichen Vorgaben leben zu lernen« (Spivak 2017, 17).

Ich würde ihre Überlegung wie folgt ausweiten: die Unschlüssigkeit zwischen dem Realen und dem Virtuellen verwirrt uns Bürger und begünstigt den opportunistischen Zynismus von Politikern, Unternehmen und einigen sozialen Bewegungen, die Groucho Marx so treffend ironisiert hat (»Meine Prinzipien sind folgende: Wenn euch das nicht gefällt, dann habe ich was anderes für euch«). Über die Annahme der Heterogenität der Widerstände komme ich noch zu einer weiteren Art, die Konflikte und die Handlungsweisen der Lateinamerikaner sogar im Fall der Dissidenz innerhalb einer Nation, eines Genderkonzepts oder einer Ethnie ohne jeglichen Totalisierungsanspruch zu verstehen. Der anfängliche Weg dahin ist bescheidener als die Annahme, es handele sich dabei um eine anthropologische Sichtweise oder um eine Epistemologie des Südens, der Dekolonialisierung oder der Technologie und ihren Alternativen. Es geht darum, auf flexible Art und Weise zu erschließen, wann diese Konflikte als Ort fungieren, an dem man sich zum Nachdenken begibt und an dem man neue, verschiedene Handlungsformen ausprobieren kann.

Eine Erneuerung der Institutionen schließe ich nicht aus. Es kann weiterhin sinnvoll sein, altbewährte Wege der zivilen Teilhabe zu nutzen, wenn es sich lohnt. Dazu zählen Abstimmungen, Bürgerhaushalte, Straßenproteste, das gezielte Nutzen der Bildschirme. Rebellionen ausgehend von bespitzelten Bürgern, Widerstandsgruppierungen und kleinere Umverteilungen der Macht, die gelegentlich im Stadtrat oder in Gremien zur regionalen Integration auftreten, sind nicht per se überholt. Aber um Formen der Staatsbürgerschaft zu erkunden, die weder von der Institutionalität, noch von den Apps und deren Gewinnorientierung eingefangen werden, liefern die unabhängigen Bewegungen mit ihren neugestalteten Strategien und ihrer Art und Weise, Menschen zu führen, eine hochinteressante Alternative. Sie beziehen sowohl kollektive, als auch individuelle Erfahrungen mit ein, und betten diese sowohl in den makro-, also auch in den mikrosozialen Kontext ein. Sie entwerfen dadurch gesellschaftliche Montagen und beziehen Stellung angesichts der Arroganz derer, die meinen, die Gesellschaft, den Sprachgebrauch 
und die Algorithmen zu steuern: Schaut her, wir haben ganz andere Fragen, und es macht uns keine Angst, dass sie widersprüchlich sind!

Durch Algorithmen ausgetauschte Bürger? Das sind keine Fake News, sondern nur eine Dimension dessen, was in der heutigen Zeit geschieht.

Mexiko-Stadt, im Juli 2019 
